\title{
Le flagellé Tricbomonas vaginalis \\ et son environnement bactérien en milieu vaginal
}

\author{
par R.-M. NICOLI, J. NOURRIT, A. MUNIGLIA et A. MICHEL-NGUYEN \\ Laboratoire de Parasitologie, Faculté de Médecine, secteur nord, bd. P.-Dramard \\ F 13326 Marseille Cedex 3
}

\begin{abstract}
RESUME. Etude statistique réalisée depuis plusieurs années sur les peuplements vaginaux. Il apparaît nettement que Trichomonas vaginalis bouleverse les peuplements bactériens en dégradant pour un temps leur équilibre initial d'où l'apparition de xénoécies inhabituelles à multiples germes puis à germe unique où se manifestent des effets purement interactifs entre des germes à virulence variable alors même qu'apparaît une sollicitation lymphokinique de l'organique, extériorisée par une diapédèse et une pyogénèse, qui peut, à certains instants, être intense.
\end{abstract}

The Flagellate Trichomonas vaginalis and its bacterial environment in vaginal medium.

SUMMARY. Statistical study on the vaginal bacterial degradations induced by the flagellate Trichomonas vaginalis

Accepté le 5 juin 1980. 
Alors que la Trichomonase uro-génitale masculine est, dans la règle, peu bruyante, la Trichomonase uro-génitale féminine est une affection cyclique aux caractères cliniques bien connus:

- phase aiguë à leucorrhée abondante, spumeuse souvent verdâtre et nauséabonde, parfois striée de sang, à granulations vaginales et irritation locale que traduisent volontiers dyspareunies et classiquement (mais inexactement) prurit (Trichomonasis acuta et même Trichomonasis culminans) lorsque le contenu vaginal n'est plus qu'une purée pyocytaire (vaginite de Hoehne).

- Phase chronique ou latente où tous les signes s'amendent mais susceptible à tout instant de revenir à la phase aiguë (rechutes) (Trichomonasis chronica).

Depuis quelques années, pour des raisons qui ne nous apparaissent pas clairement, nous observons une raréfaction notable de l'affection dont la fréquence à Marseille est passée de $25 \%$ (vers 1960-1965) à 7,5\% aujourd'hui (1974-1979) (1).

Nous avons donc observé sur 1277 prélèvements, 94 cas de Trichomonase féminine (soit $7,36 \%$ ) dont 82 trichomonases exclusives $(6,42 \%)$, c'est-à-dire environnées de germes banaux ou exceptionnellement sans cortège bactérien et 12 trichomonases associées $(0,94 \%)$ : 1 avec Neisseria gonnorhoeae, 6 avec Corynebacterium vaginale, 1 avec Peptostreptococcus anaerobius, 4 avec Candida albicans (2). Dès lors, se posait le problème encore bien méconnu de l'environnement bactérien en milieu vaginal de Trichomonas vaginalis, problème que nous avons résolu en deux temps :

1. - Etude de la fréquence des divers germes sur deux échantillons : 94 Trichomonas + et 1183 Trichomonas -

2. - Etude de l'action de Trichomonas vaginalis sur les peuplements vaginaux.

\section{1 - Trichomonas vaginalis et 1 fréquence des germes bactériens.}

Nous obtenons le tableau suivant (tableau I) et dès lors, pouvons classer les germes en trois catégories :

a) Germes dont la fréquence est inchangée (ou pratiquement inchangée en cas de Trichomonase) : Klebsiella pneumoniae et à moindre degré Staphylococcus saprophyticus.

(1) Durant le même temps, la situation de la candidose vaginale s'est inversée : les Candida (albicans essentiellement) sont devenus excessivement courants. Nous avons rapporté ce fait à l'usage très général des œstroprogestatifs contraceptifs et spécialement à l'action androgénique (glycogénopexique) des métabolites des progestagènes utilisés (R.-M. Nicoli 1978-1979).

Il est bien possible que les déséquilibres locaux inhérents à la carence hormonale induite (en œstrogènes surtout) soient en cause dans la raréfaction de la Trichomonase.

(2) On peut remarquer que, dans le contexte sexologique actuel, ce dernier chiffre paraît très faible. Nous y voyons une confirmation de l'hypothèse émise à la note précédente: vis-à-vis de la carence œstrogénique, Trichomonas vaginalis et Candida albicans réagissent différemment. 


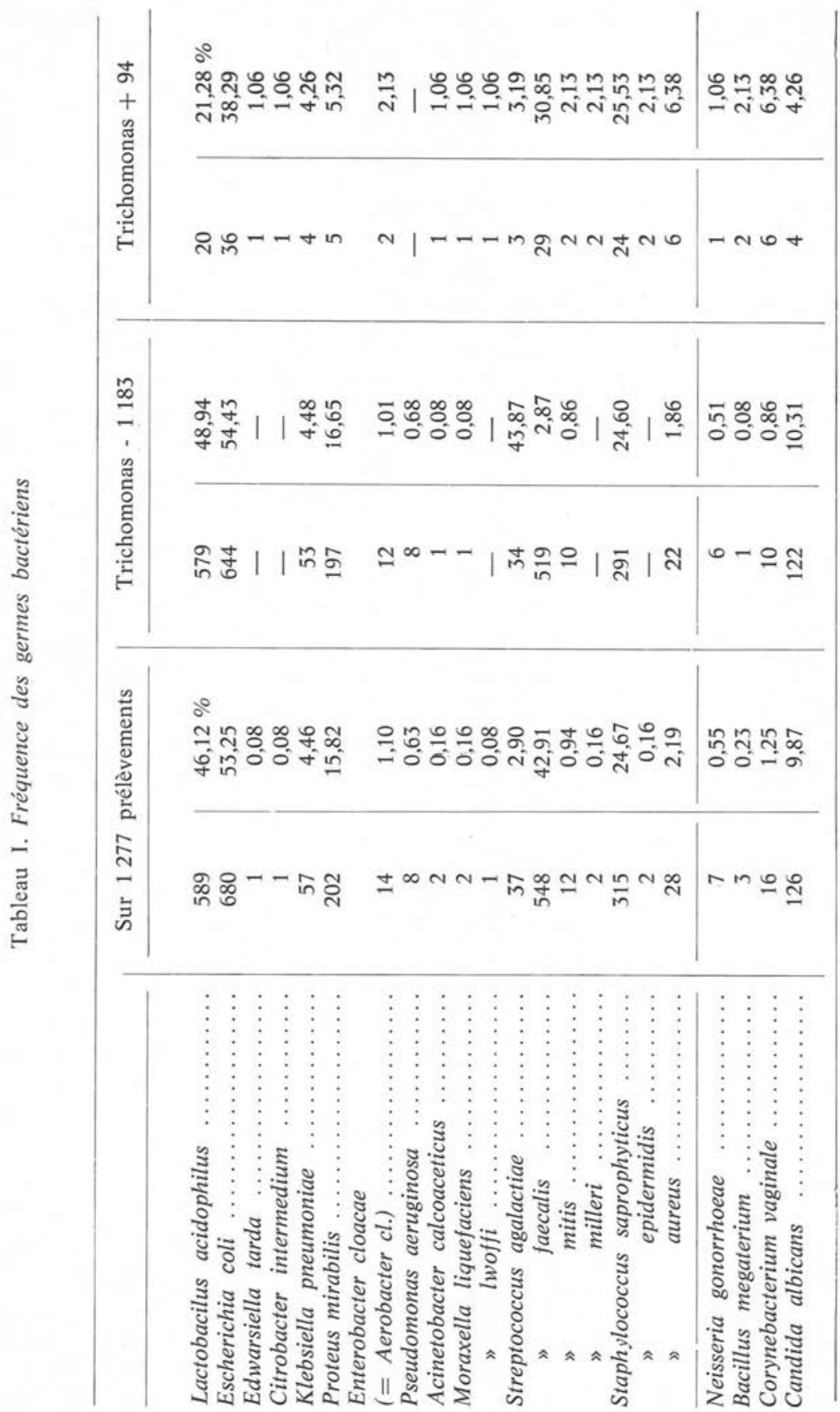


b) Germes dont la fréquence est diminuée : presque tous les germes les plus banaux :

- Lactobacillus acidophilus (le bacille de Döderlein),

- Escherichia coli (le colibacille), Proteus mirabilis, Pseudomonas aeruginosa (le pyocyanique), Streptococcus faecalis (l'entérocoque), Candida albicans.

\section{c) Germes dont la fréquence est augmentée :}

- des germes gram-négatifs : Edwarsiella tarda, Citrobacter intermedium, Aerobacter cloacae, Acinetobacter calcoaceticus, Moraxella liquefaciens et $M$. lwoffi et

- surtout des germes gram-positifs : Streptococcus agalactiae, St. mitis, St milleri, Staphylococcus épidermidis et Staphylococcus aureus, Bacillus megaterium et deux germes vénériens Neisseria gonorrhoeae (le gonocoque) et Corynebacterium vaginale (le bacille glucidolytique de Lutz), sans oublier l'interférence entre Trichomonas vaginalis et les peuplements anaérobies. Nous avons démontré naguère que le Leptothrix vaginalis des gynécologues, accompagnant fréquent de Trichomonas vaginalis, n'était en fait qu'un Sphaerophorus funduliformis en association symbiotique avec le flagellé.

\section{2 - Trichomonas vaginalis et les peuplements vaginaux.}

Cette perspective, plus synthétique qu'analytique, est beaucoup plus intéressante : elle fournit des informations capitales sur la dégradation des peuplements vaginaux en présence de Trichomonas vaginalis.

Bien évidemment, elle présuppose une schématisation allélogique (au demeurant bactério-sociologique) peu habituelle, sur laquelle nous devons tout d'abord nous arrêter.

\section{a) La notion de xénoécie.}

Nous définissons comme xénoécie un peuplement cavitaire lié à la présence d'agents divers essentiellement bactériens ou fongiques - les xénontes - peuplement relativement stable de l'enfance à la mort aux intercurrences prés, intercurrences liées à l'apport digestif pour le peuplement entérique, aux aléas sexuels ou hydriques (soins intimes) pour le peuplement vaginal.

Dans de tels cas - et c'est justement ce qui se passe avec Trichomonas vaginalis, xénonte vénérien - la xénoécie préexistante subit une dégradation au moins temporaire (durant la phase aiguë) avec apparition d'un facies lié à la multiplication du nouveau venu (3).

(3) Nous n'avons donc pas pu reprendre les concepts traditionnels en phytosociologie d'associations ou de sociations en raison de l'impossibilité où nous sommes de définir des espèces caractéristiques d'un peuplement déterminé. L'eurytopie des germes bactériens s'y oppose absolument.

Nous utilisons cependant, comme les phytosociologues, pour désigner les xénoécies, un polynome constitué par la suite des noms de genres, suivis le premier du suffixe - o - et le dernier du suffixe - etum (à la suite du radical), le vocable spécifique étant le cas échéant cité au génitif. 
Les xénoécies dépendent bien évidemment des caractères écologiques de la cavité en cause et aussi de l'existence d'interaction entre les germes: dans une cavité limitée comme un vagin, dans un médium soumis à l'influence cyclique des stéroïdes sexuels (trophicité œstrogénique, hydratation progestative, glycogénopexie androgénique), sous l'action vraisemblable de bactériocines, chaque grand groupe bactérien n'est alors représenté que par une ou rarement deux espèces.

\section{b) Xénoécies aéro-microaérophiles et anaérobies.}

Le milieu vaginal présente deux peuplements conconnitants :

- un peuplement aéro-microaérophile, d'origine stercorale (entérique au demeurant tellurique), apparaissant dans les heures qui suivent la naissance, la greffe étant facilitée par la "crise génitale du nouveau-né », actif durant toute la vie (et responsable par le fait des Lactobacillacées de l'acidification vaginale) et doué d'un thermopréférendum élevé $\left(36-37^{\circ} \mathrm{C}\right)$;

- un peuplement anaérobie, d'origine également stercorale (tellurique) mais à thermopréférendum bas (au-dessous de $20^{\circ} \mathrm{C}$ ) et qui ne se réveillera qu'à la mort de l'intéressée pour assurer partiellement la nécessaire lyse cadavérique (nécrolyse) (en association avec d'autres germes identiques, ceux-là intestinaux essentiellement).

Trichomonas vaginalis, xénonte anaérobie, dégrade les xénoécies aéro-microaérophiles, au bénéfice parfois de quelques germes du cortège anaérobie (Peptostreptococcus, Peptococcus, Ristella et surtout Sphaerophorus).

\section{c) Constitution des xénoécies aéro-microaérophiles.}

Quatre éléments bactériens, représentés par une (ou plus rarement deux) espèces se manifestent fondamentalement :

1 - une Lactobacillacée, le Lactobacille de Döderlein généralement Lactobacillus acidophilus ;

2 - un germe gram-négatif asporulé (Entérobacteriacées ou Pseudomonadacées) : Escherichia coli étant le plus commun suivi de Proteus mirabilis, de Klebsiella pneumoniae et d'Aerobacter cloacae ;

3 - une Streptococaccée, Streptococcus faecalis surtout, suivi par Streptococcus agalactiae ;

4 - une Micrococcacée du genre Staphylococcus, Staphylococcus saprophyticus le plus souvent mais aussi Staphylococcus aureus auquel la pathogénicité donne souvent l'allure d'une germe de surinfection.

De ces quatre éléments dépendent des xénoécies à 4 germes, à 3 germes, à 2 germes, à 1 germe, les xénoécies à 2 germes étant de très loin les plus nombreuses (tableau II). 
Tableau II. Les xénoécies vaginales courantes

Sur 1277 prélèvements ont été observés :

3 fois des xénoécies à 4 germes, soit $\quad 0,23 \%$;

10 fois des xénoécies à 3 germes, soit $0,78 \%$;

1220 fois des xénoécies à 2 germes, soit 95,54\%;

42 fois des xénoécies à 1 germe, soit $3,29 \%$;

2 prélèvements abactériens soit $0,16 \%$.

Nous avons ainsi reconnu, parmi 1277 prélèvements :

2 types différents de xénoécies à 4 germes

5 types différents de xénoécies à 3 germes

2 types différents de xénoécies à 2 germes

48 types différents de xénoécies à 2 germes

et à espèces multiples

11 types différents de xénoécies à 1 germe

monospécifiques.

Les xénoécies suivantes étant les plus fréquentes :

Escherichio-Streptococcetum faecalis,

Lactobacillo-Streptococcetum faecalis,

Lactobacillo-Escherichietum coli,

Escherichio-Staphylococcetum saprophytici,

Lactobacillo-Staphylococcetum saprophytici,

Escherichio-Proteetum mirabilis,

Proteo (mirabilis)-Streptococcetum faecalis,

Proteo (mirabilis)-Staphyloccetum saprophytici.

$$
\begin{aligned}
& =275 \text { soit } 21,53 \%, \\
& =194 \text { soit } 15,19 \%, \\
& =148 \text { soit } 11,59 \%, \\
& =135 \text { soit } 10,57 \%, \\
& =130 \text { soit } 10,18 \%, \\
& =55 \text { soit } 4,31 \%, \\
& =53 \text { soit } 4,15 \%, \\
& =33 \text { soit } 2,58 \% .
\end{aligned}
$$

Toutes ces xénoécies sont susceptibles de dégradation lorsque surgit un nouveau venu bactérien ou eucaryote : certains ne modifient pas ou modifient peu le peuplement initial : ainsi en est-il de la bactérie des dermatoglyphes Bacillus megaterium, du champignon Candida albicans, le plus fréquent des levures vaginales. D'autres ont une action considérable tels la bactérie parasitotrophe Neisseria gonorrhoeae et le Flagellé troglobiote Trichomonas vaginalis.

\section{d) Action de Trichomonas vaginalis sur les xénoécies initiales :}

- le faciès à Trichomonas vaginalis est un faciès variable oscillant entre l'aspect purulent de la Trichomonase aiguë avec dégradation profonde des xénoécies préexistantes et l'aspect normal de la Trichomonase chronique où se reconstitue la xénoécie initiale.

Les chiffres enregistrés par nous sont les suivants (tableau III) :

- L'analyse d'un tel tableau entraîne des conclusions immédiates : sur 15 xénoécies à 4,3 , et 2 germes multispécifiques, 11 sont liés à Trichomonas vaginalis $(73,33 \%)$, sur 1218 xénoécies à 2 germes monospécifiques, 41 seulement sont liées à Trichomonas vaginalis $(3,37 \%)$.

- Sur 42 xénoécies à 1 germe, 30 sont liées à Trichomonas vaginalis $(71,43 \%)$. 


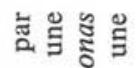

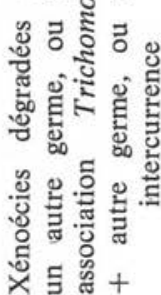

¿ㅇำ

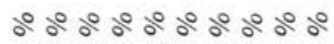

8.808

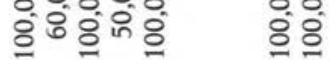

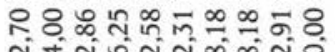

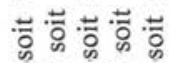

규유

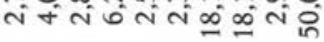

- in $-\mathrm{N}$

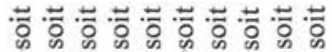

竞

- -

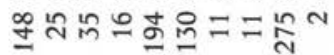

- - -m- -

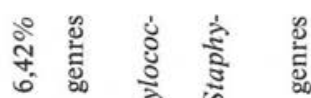

एक

压离

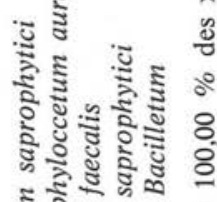

:

$\circ$ 일

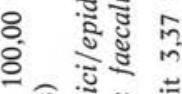

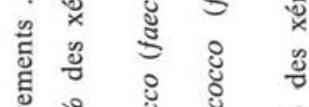

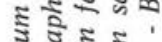

․ㅠ

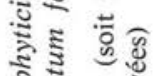

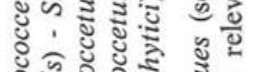

인

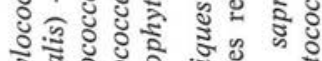

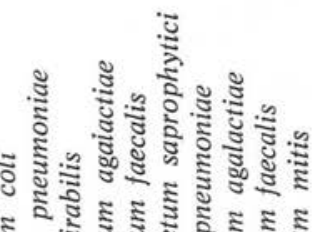

कू

की की

₹

论

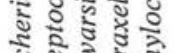

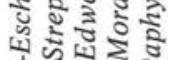

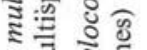

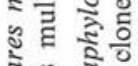

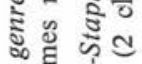

ㅇํㅇํำ

$\sim$ 离 :

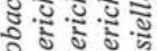

पूर

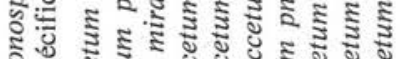

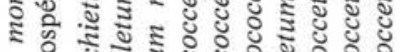

०.

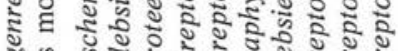

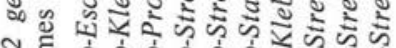

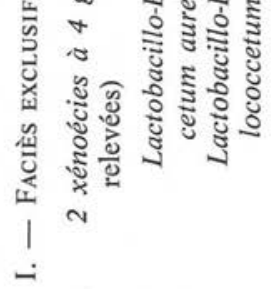

๘

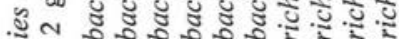


o

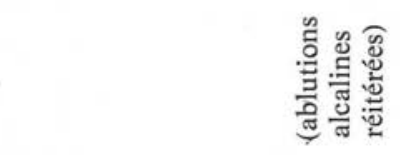

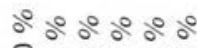

8.8888

ร.j5․․

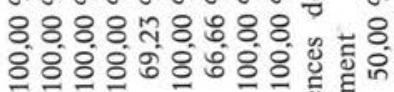

8ं

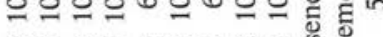

:

- 丝一篮-

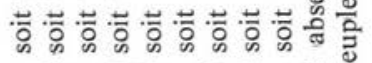

$-N-a m-a n-N \check{D}$

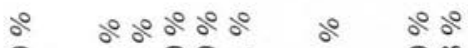

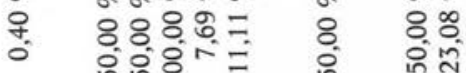

苛

少 NN-ma n NM

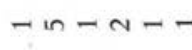

$-1-a \sigma-0 N-$

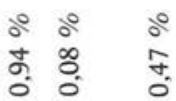

$\begin{array}{ll}0 & 0^{\circ} \\ 0 & \frac{m}{2}\end{array}$

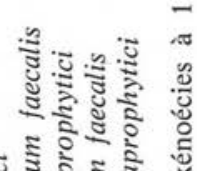

:

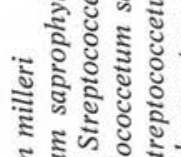

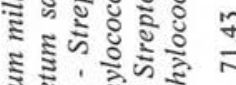

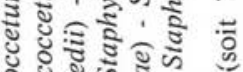

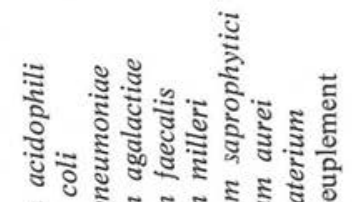

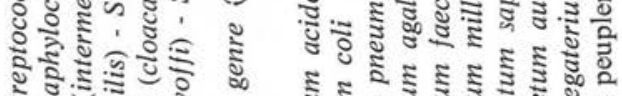

के

.

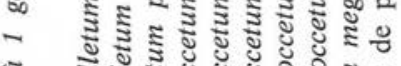

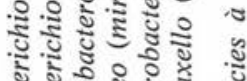

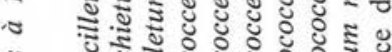

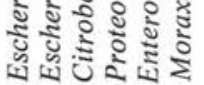

ชัญ

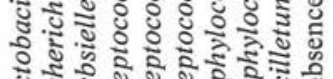

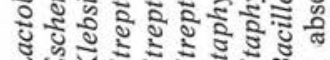

ते
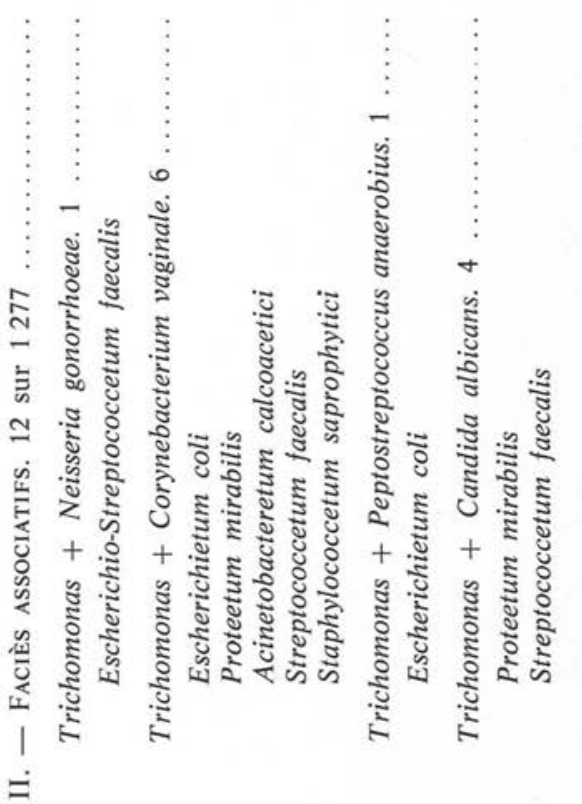
- Une telle dispersion ne saurait être liée au hasard: tout se passe comme si, dans un premier temps, la venue de Trichomonas vaginalis exacerbait des peuplements latents (apparition de 1 ou 2 types élémentaires inapparents jusque là) pour dans un second temps amener la disparition temporaire de 2 ou 3 types élémentaires, ou même l'élimination de tout peuplement apparent avant d'en arriver à une période de latence (chronicité) avec réapparition des peuplements basaux.

C'est justement ces bouleversements induits qui entraînent des ruptures d'équilibre qu'il faut bien interpréter à deux niveaux :

1) rupture de l'équilibre entre les germes eux-mêmes dont les virulences (pouvoir de multiplication) sont différents ;

2) sollicitation des facteurs lymphokiniques, d'où une diapédèse et une pyogénèse accrues alors même que, la virulence aidant, l'un des germes bactériens devient prépondérant.

Remarque: Trichomonas vaginalis est de plus recouvert d'une vêture schizophytique à Protozoobactéries anaérobies (Treponema) dont l'action est encore inconnue.

\section{Conclusion}

La Trichomonase uro-génitale s'accompagne donc d'un bouleversement profond mais temporaire ou plutôt cyclique, des peuplements bactériens cavitaires. Ce sont ces peuplements perturbés qui sont responsables des signes cliniques de l'affection, alors que le flagellé n'a qu'un rôle inducteur lié peut-être à des phénomènes de concurrence nutritionnelle ou à des déséquilibres induits par l'action d'exoactones, métabolites encore à découvrir.

\section{BIBLIOGRAPHIE}

Butler B. C., Beakley J. W. : Bacterial flora in vaginitis. Am. J. Obstet. Gynecol., 1960, 79, $433-440$. Gardner H. L., Damper T. K., Dukes C. D. : The prevalence of vaginitis. A study in incidence. Am. J. Obstet. Gynecol., 1957, 74, 1080.

Gordon A. M., Hughes H. E., Barr G.T. D. : Bacterial flora in abnormalities of the female genital tract. J. Clin. Pathol., 1966, 19, 429.

Nicoli R.-M., Fonsagrive D., Buffard Y.: Les xénoécies bactériomycéliennes du tractus urogénital féminin. Note préliminaire. Rev. Fr. Gynecol. Obstet., 1977, 72, 721-723.

Nicoli R.-M., Muniglia A.: Esquisse d’une dynamique du microbisme génital. Rev. Fr. Gynecol. Obstet. 1979, 74, 313-320.

Ritzerfeld W.: Die normale und pathologische Flora des genital traktes. Gynaecologe, 1969, 2, 2-6.

Thabaut A., Durosoir J.-L., Delacroix P., Anglade J.-P.: La flore bactérienne du vagin. A propos de 995 prélèvements. Méd. Armées, 1974, 2, 513.

Wurch Th., Lindler R.: Le concept d'association dans les biocénoses vaginales. Gynecol. Obstet., $1967,66,347-354$.

Wurch Th., Lindler R., Humbel R., Braun M.-Th.: Dynamique d'associations dans les biocénoses à Trichomonas vaginalis. Act. Anat. Pathol., 1967, 15, 252.

Wurch Th., Lutz A.: Etude du contenu vaginal dans 500 cas de leucorrhées. Cytolyse, microbiologie, Rev. Fr. Gynecol. Obstet., 1955, 50, 289. 